\title{
The physics of pot-in-pot coolers
}

Arsène Chemin, ${ }^{1}$ Victor Levy Dit Vehel, ${ }^{1}$ Aude Caussarieu, ${ }^{1}$ Nicolas Plihon, ${ }^{1}$ and Nicolas Taberlet ${ }^{1, a)}$

Univ Lyon, Ens de Lyon, Univ Claude Bernard, CNRS, Laboratoire de Physique, Département de Physique, F-69342 Lyon, France

(Dated: 14 February 2017)

A pot-in-pot cooler is an affordable electricity-free refrigerator which uses the latent heat of vaporization of water to maintain a low temperature inside an inner compartment. In this article we experimentally investigate the influence of the main physical parameters in model pot-in-pot coolers. The effect of the wind on the evaporation rate of the cooling fluid is studied in model experiments while the influence of the fluid properties (thermal conductivity, specific heat and latent heat) is elucidated using a variety of cooling fluids (water, ethanol and ether). A model based on a simplified heat conduction equation is proposed and is shown to be in good quantitative agreement with the experimental measurements.

\section{INTRODUCTION}

A pot-in-pot cooler, clay-pot cooler, or zeer, is a simple and cheap electricity-free evaporative cooling device. It consists in a porous outer pot, in which a smaller waterproof inner pot (often glazed clay) containing the foodstuff is fitted. The spacing between the pots is filled with sand and water which slowly percolates to the outer surface of the pot and evaporates (see Fig. 1). Since the vaporization of water is an endothermic process the energy required is taken from the system, causing the temperature in the inner pot to drop by typically $5^{\circ} \mathrm{C}$ to $20^{\circ} C^{1,2}$

The use of pot-in-pot coolers can be tracked to 2500 BC in Egypt and as early as 3000 BC in Indus Valley civilizations ${ }^{3}$ but interest in such affordable and simple devices has recently been renewed. ${ }^{4,5}$ Similar devices can be found in different cultures: a botijo is a traditional element of the Spanish culture and consists in a single porous clay pot, usually filled with water. It relies on the same principle although the stored fluid itself acts as the refrigerant. ${ }^{6}$ Pot-in-pot coolers are still widely used and their design and optimization is the focus of a vast technical engineering literature ( $\mathrm{see}^{7-12}$ and references therein).

The goal of this paper is to propose simple hands-on experiments to explore the physics behind the pot-in-pot cooler. In a single project-based laboratory work, students could practice modelling and grasp fundamental concepts in thermodynamics which are often adressed in separate experiments. Namely, the proposed setup is a great introduction to heat conduction ${ }^{13-15}$, to the concept of latent heat ${ }^{16,17}$, supercooling ${ }^{18}$ and can demonstrate that water can be frozen into ice through evaporation. ${ }^{19,20}$

The article is organized as follows. Section II is dedicated to the modeling of the simplified systems. The influence of the wind speed and of the nature of the liquids used are studied and a simplified governing equation is proposed. In section III experimental results on the cooling of various versions of clay-pot coolers are presented,

\footnotetext{
${ }^{a)}$ Electronic mail: nicolas.taberlet@ens-lyon.fr
}

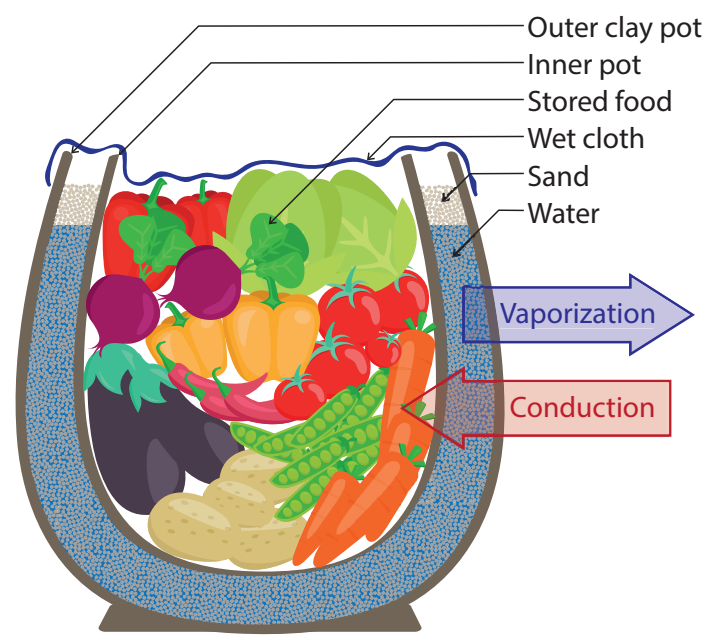

FIG. 1. Sketch of a pot-in-pot cooler. The water in the sand filling the gap between the two pots percolates through the porous outer clay pot and evaporates, cooling the entire system. Foodstuff can be safely stored inside the refrigerated inner compartment (often a glazed clay pot).

and the agreement with the theoretical predictions are discussed.

\section{MODELING}

In this paragraph, simplifying hypotheses are presented, the influence of the key parameters (size, nature of the evaporating fluid, wind) is discussed and a simplified governing equation is proposed.

\section{A. From real pot-in-pot coolers to experimental models}

Let us first discuss important parameters controlling the cooling efficiency of a real pot-in-pot cooler, as displayed in Fig. 1 and in Fig. 2a. As explained in the introduction, the matter within the pot - referred to as 
"cooled matter" in the remaining of the text - is cooled through the evaporation of a liquid stored between the inner and outer pots - referred to as "evaporating liquid" in the remaining of the text. The size of the cooler is the first important parameter: increasing the surface increases the cooling energy provided to the pot but the associated increase in total volume, and therefore total heat capacity, might counterbalance the benefits. As it is customary in heat conduction problems, the total heat capacity scales as the volume while the thermal losses scales as the surface area of the system. ${ }^{21,22}$ Moreover, in a pot-in-pot cooler, the cooling power also scales as the surface and while it initially exceeds the losses, they eventually balances out to reach an equilibrium. Therefore, at the first order, the equilibrium temperature should be independent of the size (provided that the outer pot is kept wet), whereas the cooling dynamics should be faster for smaller systems. The thickness of the pots and of the sand-layer lying in between is also an important geometric parameter. Increasing this thickness increases the thermal resistance to heat conduction, the effect of which remains unclear. Indeed, a greater resistance will help maintaining the pot cool, but it will simultaneously limit the cooling provided by the evaporation. Again, the optimal size will depend strongly on the exact conditions of use (and more specifically on the water resources available).

The role of the sand is to help prevent the inner pot from floating and to evenly distribute the water on the whole height of the pot through capillarity. In most actual pot-in-pot coolers, the inner pot is glazed to prevent any water leakage inside the cooling compartment. However, as explained in the introduction, a simpler cooling device can be used: a botijo simply consists in a unique porous clay pot in which the stored and cooled liquid also acts as the evaporating liquid (see Fig. 2b). This kind of cooler is usually used to cool water (or sometimes wine) although some of the liquid is lost.

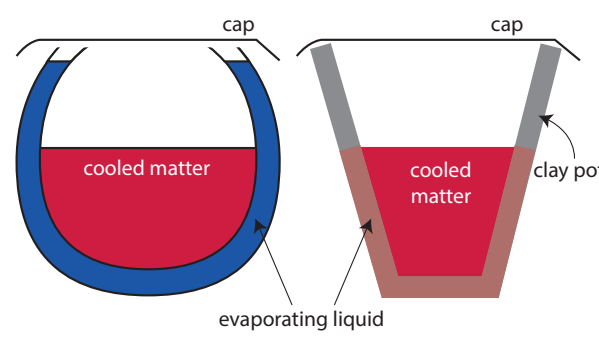

a) real pot in pot

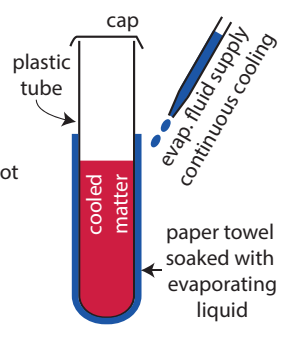

c) model pot in pot
FIG. 2. Sketches of a) a real pot-in-pot cooler, b) a botijo and c) a model pot-in-pot cooler (a test tube).

In order to simplify the system and focus on the main physical parameters, most of the experiments reported in this article were conducted in long cylindrical containers (test tubes or graduated cylinders) as displayed in the rightmost panel of Fig. 2. The cooled matter was re- stricted to liquids (water or ethanol) for simplicity. The height is noticeably greater than the diameter and as a crude approximation, the system may be considered two-dimensional. Note that the temperature gradient is mostly radial (the containers were capped) and as a consequence little convection within the fluid is expected. Our model pot-in-pot cylinder is wrapped in a thin paper towel, initially soaked in an evaporating liquid.

\section{B. Nature of the evaporating liquid}

As explained above, the origin of the cooling lies in the latent heat of evaporation of the outer liquid. During evaporation at the surface of the porous outer pot, the corresponding energy is taken both from the pot itself and the surrounding environment.

The optimization of the pot-in-pot cooler thus requires a combination of high latent heat of evaporation $L_{v}$ (of the evaporating liquid) as well as a high evaporation rate, $\dot{m}_{e}$. Table I reports the physical properties of the three liquids used (water, ethanol and ether). The exact computation of the evaporation rate is a rather complex $\operatorname{task}^{23}$, beyond the scope of the current paper, but at the first order, it follows: ${ }^{24}$

$$
\frac{\partial m_{e}}{\partial t} \propto P_{\mathrm{eq}}(1-H)
$$

where $P_{\text {eq }}$ the equilibrium vapor pressure of the evaporating liquid and $H$ its corresponding humidity (ratio of partial pressure to $P_{\mathrm{eq}}$ ). While the latent heat of water exceeds that of ethanol (by a factor 2) and ether (by a factor 6 ), the huge discrepancy in their equilibrium vapor pressure (that of ether being 25 times that of water) shows that ether is a much more efficient cooling fluid.

\begin{tabular}{|c||c|c|c|}
\hline & Water & Ethanol & Ether \\
\hline $\begin{array}{c}\text { Density } \\
\rho\left(\mathrm{kg}^{-3}\right)\end{array}$ & 1 & 0.789 & 0.713 \\
\hline $\begin{array}{c}\text { Specific heat }(398 \mathrm{~K}) \\
c_{p}\left(\mathrm{~kJ}_{\mathrm{kg}}{ }^{-1} . \mathrm{K}^{-1}\right)\end{array}$ & 4.18 & 2.44 & 2.36 \\
\hline $\begin{array}{c}\text { Latent Heat }(398 \mathrm{~K}) \\
L_{v}\left(\mathrm{~kJ} . \mathrm{kg}^{-1}\right)\end{array}$ & 2442 & 918 & 365 \\
\hline $\begin{array}{c}\text { Equilibrium vapor Pressure } \\
(373 \mathrm{~K})\end{array}$ & 2.34 & 5.8 & 58.6 \\
$P_{\mathrm{eq}}(\mathrm{kPa})$ & & \\
\hline
\end{tabular}

TABLE I. Evaporating liquid thermodynamic data from Ref. $^{25}$

\section{Influence of air flow on the evaporation rate}

A simple and efficient way to reduce the local partial pressure at the surface of the pot is to increase air advection, i.e. to increase the wind. Experiments were conducted using a Petri dish (of diameter $8 \mathrm{~cm}$ and height 


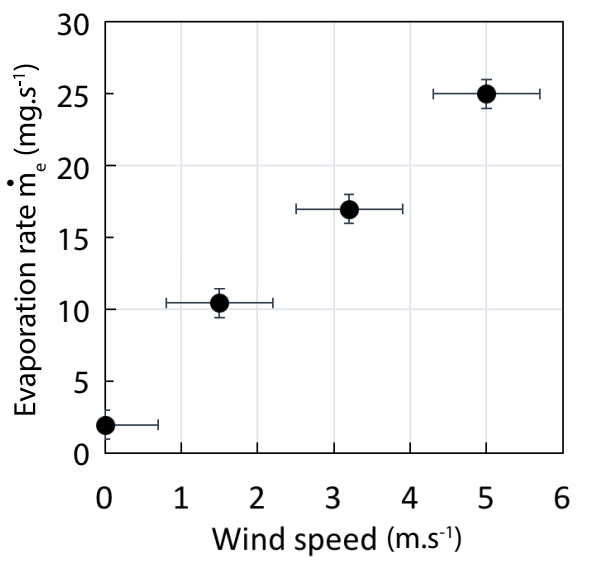

FIG. 3. Evaporation rate of water in a Petri dish $\left(50 \mathrm{~cm}^{2}\right)$ at $75 \%$ of humidity. The surface of the water is planar and the flow is plotted in terms of the surface wind speed.

$1 \mathrm{~cm}$ ) filled with water, surrounded by air at $75 \% \mathrm{hu}-$ midity and at temperature $T=23{ }^{\circ} \mathrm{C}$. Air is blown from the side of the cylinder, using a fan. Fig. 3 (a) reports the evolution of the evaporation rate (measured from the mass evolution of a the water) as a function of the wind speed measured using an anemometer. The effect of wind is crucial: the evaporation rate is multiplied by a factor ten between a situation without wind (when the partial pressure at the surface decreases mostly through molecular diffusion ${ }^{26}$ ) and a wind speed of $5 \pm 0.5 \mathrm{~m} \cdot \mathrm{s}^{-1}$ when advection dominates. The smallest air flow that the fan could produce $\left(1.5 \pm 0.2 \mathrm{~m} . \mathrm{s}^{-1}\right)$ already causes a large increase in the evaporation rate (by a factor 5 ). The wind speed therefore appears to critically affect the evaporation dynamics and actual pot-in-pot coolers are better used in a windy environment.

\section{Governing equation}

Let us first consider the heat diffusion in a long solid cylinder, of diameter $a$, height $h$, thermal conductivity $\lambda$, density $\rho$ and specific heat $c$, initially at temperature $T_{i}$, quenched to the ambient temperature $T_{0}$. The heat diffusion equation in cylindrical coordinates can be analytically solved and a simple long-term evolution of the temperature at the center of the cylinder is given in terms of series of Bessel functions of the first kind ${ }^{27}$. Letting $j_{0} \simeq 2.405$ be the first zero of the $J_{0}$ Bessel function, the dominant term reads:

$$
T-T_{0}=\left(T_{i}-T_{0}\right) e^{-t / \tau}, \text { where } \tau=\left(\frac{a}{2 j_{0}}\right)^{2} \frac{\rho c}{\lambda}
$$

Note that the temperature relaxation time may be conveniently expressed as $\tau=1 / R C$, where $C=\pi(a / 2)^{2} h \rho c$ is the total heat capacity and $R=1 /\left(j_{0}^{2} \pi \lambda h\right)$ is an effective thermal resistance. The system can therefore be seen

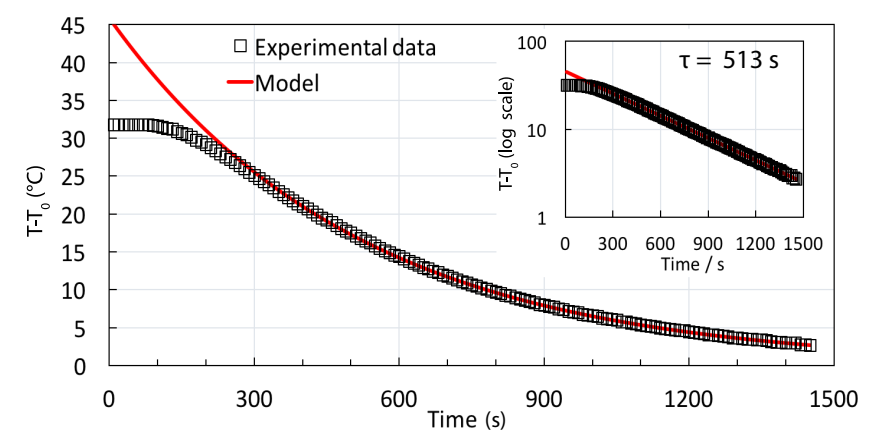

FIG. 4. Temperature evolution of a cooled PVC cylinder. Initially the cylinder is homogeneously heated at $52^{\circ} \mathrm{C}$ then placed in a $20^{\circ} \mathrm{C}$ air flow. After a transient, the temperature evolution can be described by an exponential law (see solid red line). The inset show the same data on a semi-log scale.

as a simple capacitor charging (or discharging) through a resistor.

In order to validate this approach, experimental measurements were conducted using a solid PVC cylinder (30 $\mathrm{mm}$ in diameter and $200 \mathrm{~mm}$ in height) initially heated at $52^{\circ}$ an then placed in a flow of cold $\left(T_{0}=20^{\circ}\right)$ air blowing at $3 \pm 0.3 \mathrm{~m} . \mathrm{s}^{-1}$. Obviously no convection occurs in the solid and conduction is the prevalent mode of heat transfer. A small hole was drilled along the axis of the cylinder and the temperature was recorded at its center using a thermo-couple. Fig. 4 shows the time evolution of the temperature difference $T-T_{0}$. After a transient (typically 100s), the dynamics of cooling is very well fitted by an exponential law (see red lines and insets).

Although the temperature within a solid cylinder (or in the water inside a pot-in-pot cooler) is clearly not uniform, its average value is directly linked to that at its center. Therefore, we propose to model the pot-in-pot cooler as a system of uniform temperature $T$ and heat capacity $C$, cooled at a rate given by $\dot{m}_{e} L_{v}$, where $\dot{m}_{e}$ is the evaporation rate of the evaporating liquid and $L_{v}$ its latent heat of vaporization. Energy is lost to the ambient environment at temperature $T_{0}$ through an effective thermal resistance $R$. The dynamics is then governed by:

$$
C \frac{\mathrm{d} T}{\mathrm{~d} t}=L_{v} \dot{m}_{e}-\frac{1}{R}\left(T-T_{0}\right)
$$

Note that in general $\dot{m}_{e}$ does not remain constant throughout an experiment, unless the evaporating liquid is continuously supplied to the system. The predicting power of this simplified equation is discussed throughout section III. 


\section{COOLING EXPERIMENTS}

\section{A. A real botijo}

The first experiment was conducted in a single actual clay-pot, modeling a botijo (see Fig. 2b). In this setup the cooled liquid (water) therefore also acts as the evaporating liquid. The clay-pot has a $3 \mathrm{~L}$ volume, a height $h=20 \mathrm{~cm}$, an upper diameter of $15 \mathrm{~cm}$, while its walls are $5 \mathrm{~mm}$ thick and its mass is $1554 \mathrm{~g}$. The pot is filled with $2 \mathrm{~L}$ of water. The total heat capacity of the system $\left(C_{\mathrm{p}}=9900 \mathrm{~J} / \mathrm{K}\right)$ is simply computed as the sum of that of the water and of the clay. During all the measurements, a fan was used to create a constant wind $\left(10 \pm 2 \mathrm{~m} . \mathrm{s}^{-1}\right)$.

The humidity was measured using a hygrometer and remained between 72 and $74 \%$ during the whole experiment. The mass is recorded using a weight scale (see Fig. 5(a)) and the inner temperature is measured using a thermocouple placed at the center of the pot (Fig. 5(b)) while the ambient temperature $T_{0}$ remains constant $\left(21^{\circ}\right)$ during the experiments. In this experiment with a constant ventilation acting on the pot, and a virtually infinite amount of evaporating liquid, the overall mass decreases linearly, indicating a constant evaporation rate, $\dot{m}_{e}=-41 \pm 5 \mathrm{~g} \cdot \mathrm{h}^{-1}$ given by the linear fit shown in Fig. 5(a).

The temperature of the water gradually decreases from $21^{\circ} \mathrm{C}$ to $15.3{ }^{\circ} \mathrm{C}$ over the course of four hours and then stabilizes. This corresponds to an equilibrium between the negative energy gain due to the evaporating water and the positive conductive heat flow from the warmer ambient air through the pot.

The solid red line on Fig. 5(b) represents the theoretical prediction of the evolution of the temperature calculated from Eq. 3 considering a constant evaporation rate:

$$
\left\{\begin{array}{l}
T=T_{e q}+\left(T_{0}-T_{e q}\right) e^{-t / R C} \\
T_{e q}=T_{0}+R L_{v} \dot{m}_{e}
\end{array}\right.
$$

The value of the effective thermal resistance can be computed from the equilibrium temperature $T_{e q}$ and the previously fitted evaporation rate $\dot{m}_{e}$ as:

$$
\frac{1}{R_{f i t}}=\frac{L_{v} \dot{m}_{e}}{T_{0}-T_{e q}}=4.7 \pm 0.5 \mathrm{~W} . \mathrm{K}^{-1} .
$$

The agreement between the theory and the experimental measurement is very satisfactory. Indeed, with no further fitting parameter, the characteristic decay time is well predicted. The agreement is all the more satisfactory that roughly $15 \%$ of the water has evaporated over the duration of the experiment, changing the total heat capacity.

Note that the fitted value of the effective thermal resistance can be compared to that of a long water cylinder
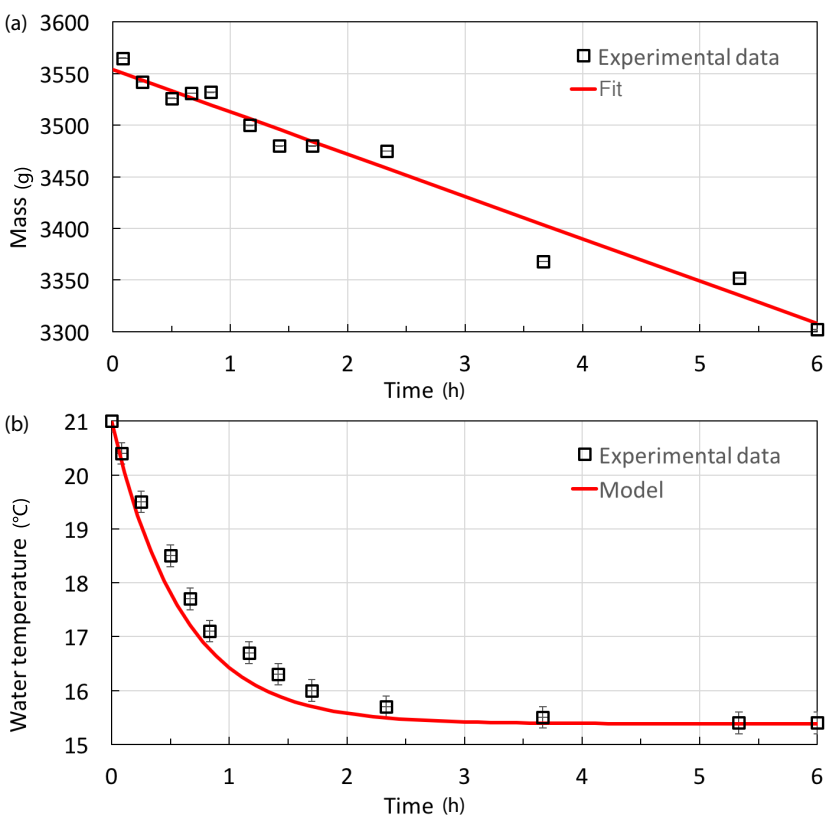

FIG. 5. (a) Time evolution of the the mass of the evaporating liquid (water). The solid red line is a linear fit, corresponding to a constant evaporation rate $\dot{m}_{e}=-41 \mathrm{~g} \cdot \mathrm{h}^{-1}$. (b) Temperature evolution of the water measured at the center of the clay pot. The solid red line is the prediction of Eq. 4

given in section II.D, using only the thermal conductivity of water $\lambda_{w}$

$$
\frac{1}{R_{\text {cylinder }}}=5.76 \pi \lambda_{w} h \simeq 2.2 \pm 0.1 \mathrm{~W} . \mathrm{K}^{-1} .
$$

The values are of the same order of magnitude but differ significantly. Indeed, the clay pot used is far from a 2D system and the surface area of the bottom and the top of the container should be taken into account: the measured resistance is thus considerably less than that of a corresponding infinite cylinder.

\section{B. Model pot-in-pot cooler}

A model pot-in-pot cooler, with a limited amount of evaporating liquid, consisting in a plastic test tube, is studied in this section.

\section{Experimental details}

The test tube has a diameter of $30 \mathrm{~mm}$, a height of $90 \mathrm{~mm}$, while the walls are $1 \mathrm{~mm}$-thick, and it is filled with $50 \mathrm{~mL}$ of distilled water. The contribution of the container itself is therefore neglected in the total heat capacity of the system and in the effective thermal resistance. The test tube is wrapped in a paper towel, initially soaked with $m_{e 0}=5 \mathrm{~g}$ of ethanol, acting as the evaporating liquid. The model pot-in-pot is placed 
vertically in a constant air flow of $3.2 \pm 0.3 \mathrm{~m} . \mathrm{s}^{-1}$ created by a fan, and as discussed above, the partial pressure of ethanol in the environment remains negligible throughout the experiment. The time evolution of ethanol mass is recorded by a weight scale and is plotted in Fig. 6(a).

\section{Evaporation rate}

Contrary to the previous experiment where the amount of evaporating liquid was virtually unlimited, the evaporation rate of ethanol, $\dot{m}_{e}$ is not constant but decays exponentially (solid red line in Fig. 6(a)).

A rudimentary first-order explanation of the exponential decay of the evaporation rate can be given. If, as a crude assumption, it is assumed that the ethanol percolates to the bottom so that the wet surface of the paper towel is proportional to the amount of remaining ethanol, then (assuming a constant evaporation rate per unit of wet surface) the amount of ethanol decreases exponentially (and so does the evaporation rate). Thus the time evolution of the mass of ethanol is given by an exponential law:

$$
m_{e}(t)=m_{e 0} e^{-t / \tau_{\dot{m}}},
$$

where the characteristic time, $\tau_{\dot{m}}$, depends on the speed of wind, on the nature of the evaporating liquid used, and on the dimensions of the test tube. For the experimental results reported in Fig. 6, $\tau_{\dot{m}} \sim 250 \pm 10 \mathrm{~s}$.

Note that the mass decay reported in Fig. 6(a) is compatible with an exponential decay only over the first $500 \mathrm{~s}$ of the experiment. Discrepancies at later time are due to condensation of water vapor contained in the surrounding air onto the ethanol-wet paper towel. However, the discrepancy is small and occurs only after the evaporation rate is already negligible.

\section{Temperature evolution}

Fig. 6(b) represents the time evolution of the temperature measured using a thermocouple at the center of the test tube (black squares). Initially, due to the high evaporation rate, the temperature drops rapidly. As the temperature decreases, the heat loss to the ambient air increases while the cooling power decreases, which leads to a minimum temperature (which does not coincide with the arrest of the evaporation). Over longer times $(t>800 \mathrm{~s})$ the evaporation rate can be considered null and the system simply warms up in the surrounding air. The temperature therefore exponentially relaxes to the ambient temperature, as can be seen on the inset in Fig. 6(b). Not only does this confirm the exponential relaxation but it also allows one to precisely measure the characteristic relaxation time $\tau_{R C}$.

\section{Prediction}

To summarize, the evaporation rate of ethanol $\dot{m}_{e}(t)$ is known, the total heat capacity $C$ is computed from the amount of water used, and the characteristic time $\tau_{R C}$ linked to the effective thermal resistance is fitted on the
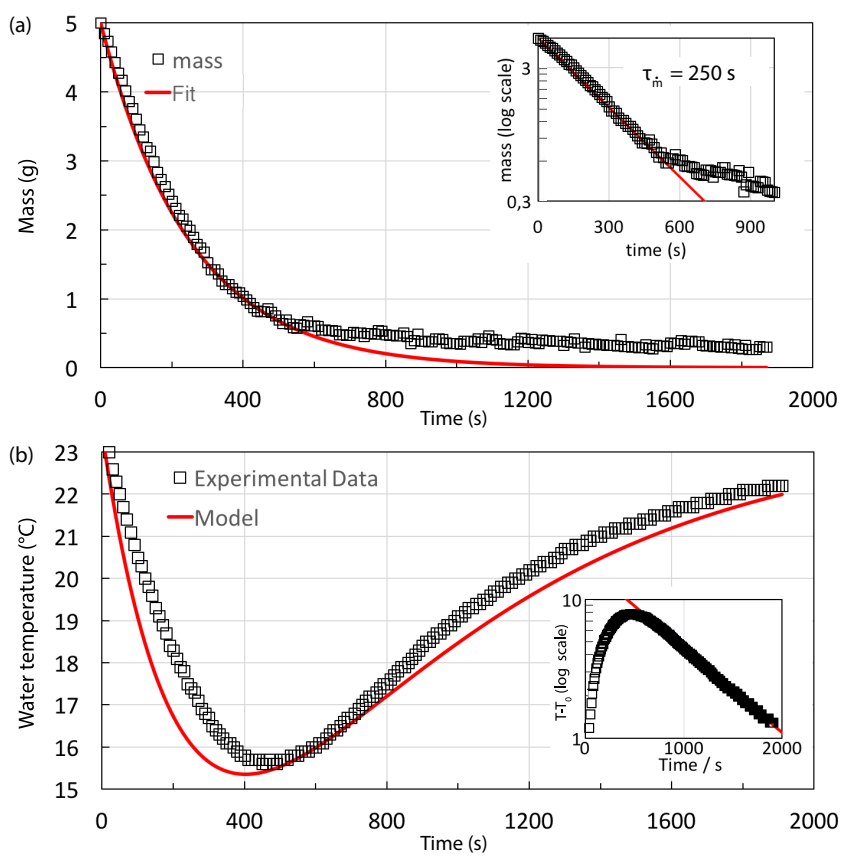

FIG. 6. (a) Time evolution of the the mass of evaporating liquid (ethanol) and exponential fit (solid red lines). The inset shows the data on a semi-log scale. (b) Temperature evolution of the water (at the center of the test tube) and model (solid red line). The inset shows the data on a semi$\log$ scale and the red line indicates an exponential fit of the final regime.

final relaxation of the temperature. Eq. 3 can therefore be solved and yields:

$$
T(t)=T_{0}-\frac{L_{v} m_{0}}{C} \frac{\tau_{R C}}{\tau_{R C}-\tau_{\dot{m}}}\left(e^{-t / \tau_{\dot{m}}}-e^{-t / \tau_{R C}}\right)
$$

This empirical/analytical solution is shown as a red line in Fig. 6(b) and shows a good overall agreement with the experimental data. Let us emphasize again that the value of the thermal resistance was computed during the final relaxation regime in which all the ethanol has evaporated. The initial cooling regime is decently fitted while the minimum temperature (and the time at which it is reached) is very well predicted. Again, one can conclude that a complex system such as a pot-in-pot cooler is accurately governed by the simplified governing Eq. 3 .

\section{Continuous cooling}

In this paragraph, a small quantity of water $(9 \mathrm{~mL})$ placed in a thin plastic test tube $(5 \mathrm{~mm}$ in diameter and $50 \mathrm{~mm}$ in height) and wrapped in a wet paper towel, is cooled by evaporating ether.

Experimental details 


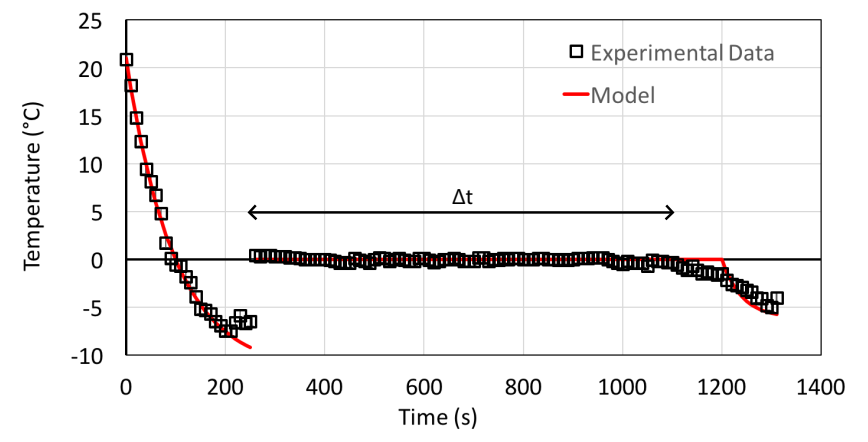

FIG. 7. (a) Temperature evolution of $9 \mathrm{~mL}$ of water in a narrow tube wrapped in a paper towel, continuously soaked with ether (added at room temperature). The water rapidly cools down into a supercooled state before it freezes (at $t=$ $240 \pm 5 \mathrm{~s})$. The cooling resumes once the liquid water has entirely turned to ice (at $t=1100 \pm 100 \mathrm{~s}$ ). The solid red line corresponds to the solution of the governing Eq. 3 .

Ether is constantly added to the system (using a pipette) as it evaporates on the paper towel. The test tube is submitted to a strong wind using a compressed air gun $(10 \pm 2 \mathrm{~m} / \mathrm{s})$ in order to further increase the evaporation rate and the experiments were conducted in a fume hood. However, the wind also has counterproductive consequences since a portion of the ether, $1-\phi$ (estimated below) is blown away before being able to play its role as evaporating liquid. In this experiment, $\dot{m}_{e}$ is directly measured from the volume released by the pipette. A further limitation is that the ether is added at room temperature, which constitutes a source of energy and tends to warm up the water stored in the tube. Finally, it should be mentioned that in such a small test tube, the effect of the temperature probe itself might not be neglected. Not only can it significantly contribute to the total heat capacity, but also acts as a radiator, conducting heat from the ambient air. The thermocouple consisted in a thin cable (the metal part has a diameter of $0.5 \mathrm{~mm}, 1.7 \mathrm{~mm}$ with the plastic insulator) but it was clear that the temperature in its direct vicinity was higher than in the rest of the fluid since it was there that the water froze last.

\section{Experimental results}

Fig. 7 shows the time evolution of the water temperature. Initially the temperature drops quickly at the rate of 1 degree every $3 \mathrm{~s}$, and within two minutes, it becomes negative indicating that the water is in a supercooled state. When the temperature reaches $-7^{\circ} \mathrm{C}$ the water abruptly starts to freeze into ice. It takes roughly $1000 \mathrm{~s}$ for the entire water to turn into ice, after which the cooling resumes.

As explained above, only a (large) fraction $\phi$ of the ether is used as a cooling fluid This fraction can be computed by fitting the first regime in which liquid water cools down to $-7^{\circ} C$, which yields $\phi \simeq 78 \pm 5 \%$, meaning that $22 \pm 5 \%$ of the ether is lost.

Interestingly, one can predict the duration of the freezing plateau, $\Delta t$, knowing the latent heat of vaporization of ether $L_{v}$, the mass of water, $m_{w}$, and its latent heat of solidification, $L_{s}$, and taking into account the temperature of the supercooled water, $T f=-7^{\circ} \mathrm{C}$ :

$$
\phi \dot{m}_{e} L_{v} \Delta t=m_{W} L_{s}+C T_{f},
$$

which yields: $\Delta t=780 \pm 100 \mathrm{~s}$. Again, the predicted duration coincides well with the experimental data since the duration of the freezing plateau is $1100-240=860 \pm 100 s$.

\section{Discussion}

Finally, we need to mention that the final regime in which the ice cools down is not very well predicted by the governing equation. According to our simplified model, there should be a clear kink in the temperature and a sharper initial decrease. Indeed, the heat capacity of ice is typically half that of liquid water while its thermal conductivity is 3 to 4 times higher. The characteristic cooling time of ice should therefore be much shorter $(6$ to 8 times) than that of liquid water, which is in clear contradiction with the experimental data. The origin of this discrepancy can be attributed to the formation of frost (condensed from the humidity of the ambient air) on the paper towel, drastically reducing the effectiveness of the evaporative cooling.

\section{CONCLUSION}

This paper shows that the complex balance between evaporation cooling and conduction heating of a system in an asymmetric air flow can actually be very well approximated by an elementary model, involving very few parameters. Provided the evaporating liquid does not contribute significantly to the heat capacity of the system, we can model the whole system as a solid object in a environment at constant temperature, with the cooling simply derived from its small mass loss. This model was verified with a very basic experimental setup, further showing the robustness of the model. The same setup can explore past the limitations of the model as well, in the case of a phase transition for instance, as shown in the last section.

\section{ACKNOWLEDGMENTS}

The authors acknowledge support from the PALSE program of the University of Lyon Saint-Etienne, the University Lyon Claude Bernard, the École Normale Supérieure de Lyon and its Physics Department and Laboratoire de Physique. We are thankful to A. Bourges, A. Guittonneau, C. Gouiller, C. Malciu, J. Sautel, B. Guiselin and E. Jaupart for fruitful discussions. 
${ }^{1}$ W. Breslyn, "Clay pot refrigerators," The Science Teacher, vol. 74 , no. 8 , p. $74,2007$.

${ }^{2} \mathrm{E}$. Anyanwu, "Design and measured performance of a porous evaporative cooler for preservation of fruits and vegetables," Energy conversion and management, vol. 45, no. 13, pp. 21872195, 2004.

${ }^{3}$ G. Dales, J. M. Kenoyer, and L. Alcock, Excavations at Mohenjo Daro, Pakistan, vol. 1. UPenn Museum of Archaeology, 1986.

4 "Ancient technology preserves food." http://www.rolexawards . com/profiles/laureates/mohammed_bah_abba/project.

5 "Food cooling system." http://content.time.com/time/ specials/packages/article/0, 28804, 1936165_1936254_ $1936632,00 . \mathrm{html}$.

${ }^{6}$ J. I. Zubizarreta and G. Pinto, "An ancient method for cooling water explained by mass and heat transfer," Chemical Engineering Education, vol. 29, pp. 96-96, 1995.

7 H. Harish and Y. K. Gowda, "Thermal analysis of clay pot in pot refrigerator," International Journal of Modern Engineering Research, vol. 4, pp. 50-55, 2014.

8 A. Naik and V. Dhir, "Forced flow evaporative cooling of a volumetrically heated porous layer," International Journal of Heat and Mass Transfer, vol. 25, no. 4, pp. 541-552, 1982.

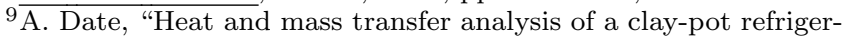
ator," International Journal of Heat and Mass Transfer, vol. 55, no. 15, pp. 3977-3983, 2012.

${ }^{10}$ R. Damle and A. Date, "Heat and mass transfer in a clay-pot refrigerator: Analysis revisited," Journal of Energy, vol. 37, pp. 1125, 2015

${ }^{11}$ R. Pandey and B. Pesala, "Heat and mass transfer analysis of a pot-in-pot refrigerator using reynolds flow model," Journal of Thermal Science and Engineering Applications, vol. 8, no. 3, p. 031006, 2016.

${ }^{12}$ A. lal Basediya, D. Samuel, and V. Beera, "Evaporative cooling system for storage of fruits and vegetables-a review," Journal of food science and technology, vol. 50, no. 3, pp. 429-442, 2013.

13 A. Díaz-Guilera, "On heat conduction in one-dimensional solids," American Journal of Physics, vol. 58, no. 8, pp. 779-780, 1990.

${ }_{14}$ M. A. Karls and J. E. Scherschel, "Modeling heat flow in a thermos," American Journal of Physics, vol. 71, no. 7, pp. 678-683, 2003.

${ }^{15}$ M. Sullivan, B. Thompson, and A. Williamson, "An experiment on the dynamics of thermal diffusion," American Journal of Physics, vol. 76, no. 7, pp. 637-642, 2008.

${ }_{16}$ M. J. Arvin, "Latent heat of vaporization experiment," American Journal of Physics, vol. 20, no. 5, 1952.

17 G. L. Salinger, "Measurement of the heat of vaporization of a liquid," American Journal of Physics, vol. 49, no. 3, 1981.

${ }^{18}$ K.-C. Tan, W. Ho, J. Katz, and S.-J. Feng, "A study of the occurrence of supercooling of water," American Journal of Physics, vol. 84, no. 4, pp. 293-300, 2016

${ }^{19}$ H. Kruglak and P. M. Loofboro, "Freezing water by evaporation," American Journal of Physics, vol. 12, no. 1, 1944.

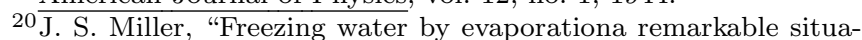
tion," American Journal of Physics, vol. 18, no. 4, 1950.

${ }^{21}$ A. C. Rowat, N. N. Sinha, P. M. Sörensen, O. Campàs, P. Castells, D. Rosenberg, M. P. Brenner, and D. A. Weitz, "The kitchen as a physics classroom," Physics Education, vol. 49, no. 5, p. $512,2014$.

${ }^{22}$ E. A. Olszewski, "From baking a cake to solving the diffusion equation," American journal of physics, vol. 74, no. 6, pp. 502$509,2006$.

${ }^{23}$ M. Branca and I. Soletta, "A physical model to help explain evaporation," The Physics Teacher, vol. 52, no. 4, 2014.

${ }^{24} \mathrm{P}$. Atkins, The laws of thermodynamics. Oxford University Press, 2010.

${ }^{25}$ W. Haynes, CRC Handbook of Chemistry and Physics, 93rd Edition. CRC Handbook of Chemistry and Physics, Taylor \&

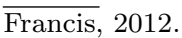

${ }^{26}$ A. J. Abraham and C. P. Bean, "A simple method for measurement of the diffusion of vapors," American Journal of Physics, vol. 57, no. 4, 1989.

${ }^{27}$ M. N. Ozisik, Heat conduction. John Wiley \& Sons, 1993. 